\title{
Avaliação de Treinamento a Distância: Reação, Suporte à Transferência e Impactos no Trabalho
}

\author{
Renata Silveira Carvalho \\ Gardênia Abbad
}

\begin{abstract}
RESUMO
O uso da Internet em treinamentos a distância vem sendo enfatizado, porém se percebem poucas avaliações sistemáticas dessas intervenções. A presente pesquisa avalia modelos de predição de três variáveis critério: Escores de Aprendizagem e Impacto do Treinamento no Trabalho, medido em Profundidade e em Amplitude. As variáveis antecedentes dos modelos integram os componentes de Reação, Características da Clientela e Suporte à Transferência. Foi avaliado um curso a distância, mediado pela Internet, oferecido gratuitamente em nível nacional. A coleta de dados foi realizada por questionários digitalizados, hospedados em páginas da Internet. Os arquivos de dados utilizados tiveram variação de 340 a 1.659 casos. Realizaram-se análises de regressão múltipla padrão e stepwise. O modelo de predição de Escores de Aprendizagem indica influência das variáveis de uso de ferramentas de interação e Reação a Resultados e Aplicabilidade. Os modelos de investigação de Impactos no Trabalho revelam relação positiva com indivíduos que percebem mais favoravelmente os resultados e a aplicabilidade do curso, relatam não haver suporte à transferência e têm elaborado um plano de negócio. São discutidas as limitações do estudo, suas contribuições e implicações práticas e teóricas.
\end{abstract}

Palavras-chave: avaliação de treinamento via Internet; reação; suporte e restrições à transferência de treinamento; impacto do treinamento no trabalho; empreendedorismo.

\begin{abstract}
The use of Internet in distance trainings is being emphasized although systematic evaluations of these interventions are barely known. The present research evaluates prediction models of three criterion variables: Learning scores and Training Impact at Work (in depth and in extent). The antecedent variables of the models integrate the following components: Reactions, Trainee Characteristics and Transfer Support. A distance training course based on Internet and nationally offered free of costs was evaluated. Data were captured with digitalized questionnaires, hosted in Internet pages. The database files varied from 340 to 1659 cases. The techniques chosen were multiple regression analyses, with enter and stepwise methods. The prediction model of Learning Scores show an influence of variables such as the use of interaction tools and Reactions to Results and Applicability. The investigation models of Impacts at Work indicate that better results in the criterion variables are obtained by individuals who perceive more positively the results and the applicability of the course, who indicate the lack of transfer support and who have elaborated a business plan. The present research discusses the limitations of the study as well as its practical and theoretical contributions and implications.
\end{abstract}

Key words: Internet based training evaluation; course reactions; support and constraints to training transfer; training impact at work; entrepreneurship. 


\section{INTRODUÇÃO}

O mundo do trabalho modificou-se nas últimas décadas. As exigências atuais são por constante aperfeiçoamento técnico e político, em que o indivíduo precisa garantir sua empregabilidade na busca incessante por atualização de habilidades, atitudes e novos conhecimentos, os quais têm prazo de validade (Meister, 1999) cada vez menor.

Paralelamente, as ações educacionais corporativas têm feito uso das novas tecnologias, em particular, a Internet. No entanto percebe-se que as novas mídias exigem recursos distintos dos utilizados em treinamentos presenciais, havendo pouco embasamento teórico e pouca preocupação em produzir conhecimento científico em treinamento a distância (Salas \& Cannon-Bowers, 2001).

Nos processos de Treinamento, Desenvolvimento e Educação - TD\&E, o uso de recursos multimídia tem crescido de forma significativa, principalmente pela possibilidade de acesso simultâneo a muitas pessoas distantes fisicamente. Diversas organizações, até mesmo as educacionais, perceberam a Educação a Distância - EaD como meio eficaz de ensino, em que custos poderiam ser reduzidos sem prejudicar a aquisição dos conhecimentos.

Em geral, as definições colocam a EaD pelo que não é em relação ao modelo presencial, mostrando apenas suas diferenças, destacando principalmente a distância física e a ausência da sala de aula. Para Belloni (1999), no entanto, a separação de tempo parece ser mais importante que a distância física entre aluno e professor.

A aprendizagem aberta, termo também utilizado, é caracterizada pela flexibilidade e autonomia do aluno, porém sem priorizar a produção de materiais e a organização industrial. A distinção entre aprendizagem aberta e EaD está no fato de que a primeira enfoca processos de aprendizagem, ou seja, a ênfase está no aluno. Já a $\mathrm{EaD}$ dá preferência aos processos de ensino, como a elaboração de materiais e definição de procedimentos instrucionais (Belloni, 1999; Maggio, 2001). Sugere-se que, para a EaD ser considerada um novo sistema educacional, as noções de autonomia e flexibilidade devam estar presentes na concepção de programas instrucionais. As concepções do conceito de aprendizagem aberta seriam, então, agregadas à $\mathrm{EaD}$.

Os conceitos aqui apresentados têm mostrado semelhanças e sobreposições, talvez não prejudiciais, o que indica que as fronteiras conceituais podem estar-se 
diluindo. Talvez não seja mesmo necessário delimitar tão claramente a distinção entre educação e uma ação de treinamento, por exemplo. Um evento instrucional visa, em última instância, a promover a educação dos colaboradores da organização. E o meio utilizado pode ser o mais criativo, desde que esteja em consonância com o objetivo de ensino.

Busca-se aqui investigar os resultados de cursos a distância, via Internet, nos níveis de satisfação do aluno, aprendizagem e impacto na sua vida profissional. Ao mesmo tempo, procura-se estabelecer relações entre os níveis de avaliação, a fim de identificar variáveis que expliquem o uso do conteúdo aprendido no contexto de trabalho.

\section{Avaliação de Treinamento: Breve Revisão da Literatura}

O foco deste estudo é a avaliação de treinamentos, em particular daqueles oferecidos na mídia Internet. Antes de abordar especificamente o núcleo conceitual do trabalho, é interessante apresentar sucintamente a classificação da avaliação de treinamento dentro do paradigma sistêmico, em que a avaliação faz parte de um sistema maior denominado Treinamento. Borges-Andrade (1997) delimita o treinamento como sistema composto por três subsistemas, a saber: levantamento de necessidades, planejamento e execução e avaliação do treinamento. $\mathrm{O}$ subsistema avaliação fornece a retroalimentação para todo o sistema, indicando os pontos positivos que devem permanecer nos treinamentos futuros, bem como sinalizando aspectos a serem modificados ou retirados do planejamento.

No contexto das ações de treinamento, as modernas tecnologias utilizadas na $\mathrm{EaD}$ trazem um novo desafio para a área de TD\&E. O hipertexto, a interatividade são exemplos dos novos elementos que ainda precisam ser muito estudados em termos de sua utilidade, efetividade e aplicabilidade real nos programas instrucionais. Para se verificar os efeitos de um evento a distância, são necessárias novas ferramentas de avaliação.

A tendência atual de se medir o efeito de um alto investimento em TD\&E vem ao encontro da percepção do valor estratégico da qualificação constante da mãode-obra de uma organização (Borges-Andrade, 2002). Para Salas e CannonBowers (2001), os avanços são teóricos, metodológicos, empíricos e práticos. Entretanto, em se falando do uso de tecnologias em eventos instrucionais, não foram encontrados resultados empíricos sobre, por exemplo, qual a natureza e o nível de interação necessária de treinandos e instrutores. 
A sistematização dos processos de avaliação tem sido interesse de vários autores que elaboraram modelos de avaliação. O modelo clássico de Kirkpatrick (1976), posteriormente modificado por Hamblin (1978), tem como preocupação central avaliar os resultados de um evento instrucional. Os níveis avaliados são de reação (satisfação do treinando com o treinamento), aprendizagem (cumprimento dos objetivos instrucionais), comportamento no cargo (aplicação dos CHAs aprendidos - Conhecimentos, Habilidades e Atitudes) e resultados, subdividido em organização (mudança nos processos organizacionais) e valor final (efeitos nos indicadores financeiros). Os autores entendem que os níveis possuem relação linear, seqüencial e têm correlação alta entre eles. No entanto as pesquisas empíricas, nacionais e estrangeiras, desde o artigo de Alliger e Janak (1989), têm chegado a resultados diferentes, não encontrando relações diretas entre os níveis.

Antes mesmo de surgirem esses questionamentos, houve a preocupação em se avaliar modelos de relação entre variáveis outras que não as relativas puramente aos resultados do treinamento. No Brasil, Borges-Andrade (1982) foi o precursor nesse sentido, propondo um modelo que inclui variáveis do ambiente como fatores influentes importantes em todo o processo de treinamento, desde o levantamento de necessidades até os resultados a longo prazo. O Modelo de Avaliação Integrado e Somativo, MAIS, é composto essencialmente por 5 componentes (insumos, procedimentos, processo, resultados e ambiente); o último subdivide-se em 4 subcomponentes: avaliação de necessidades, disseminação, apoio e resultados a longo prazo. Este modelo tem servido de base para muitas intervenções atuais, aplicadas e de pesquisa, em diversos contextos organizacionais. Este estudo apóiase parcialmente nesse modelo de avaliação, no que se refere à classificação das variáveis estudadas.

Abbad (1999), recebendo influência destes e de outros modelos de avaliação, organizou os achados da literatura em avaliação de treinamento e sugeriu um modelo de predição do impacto do treinamento no trabalho. O Modelo Integrado de Avaliação do Impacto do Treinamento no Trabalho - IMPACT é composto por sete componentes: suporte organizacional, características do treinamento, características da clientela, reações, aprendizagem, suporte à transferência e impacto do treinamento no trabalho.

Os seis primeiros componentes são considerados preditores do impacto do treinamento no trabalho. A variável critério desse modelo, impacto do treinamento no trabalho, é definida como a avaliação do treinando sobre os efeitos produzidos pelo treinamento em níveis de desempenho, motivação, autoconfiança e abertura a mudanças nos processos de trabalho.

Este trabalho baseou-se essencialmente nos modelos MAIS e IMPACT, como norteadores das variáveis a serem estudadas e do estabelecimento de relações 
entre elas. Dentre as variáveis preditoras de impacto do treinamento no trabalho, apresentam-se discussões e resultados empíricos sobre: Características da Clientela, Características do Treinamento, Reação e Aprendizagem e Suporte à Transferência de Treinamento.

Antes de iniciar a explanação sobre cada linha de pesquisa, note-se que a revisão da literatura foi realizada por meio da análise das revisões já feitas em treinamento e desenvolvimento (Abbad, 1996; Abbad, Pilati, \& Pantoja, 2003; Borges-Andrade, Salas, \& Cannon-Bowers, 2001; Tannenbaum \& Yukl, 1992), além da busca em bases de dados eletrônicas (Proquest e Web of Science) de artigos recentes envolvendo os temas aqui estudados. As palavras-chave utilizadas nas buscas foram: distance training, distance education, evaluation, instructional design, e-learning.

A primeira linha de pesquisa, seguindo o modelo de Abbad (1999), refere-se às características da clientela. Abbad et al. (2003), em revisão de literatura sobre avaliação de treinamento, apresentam as principais variáveis preditoras de impacto nas diversas linhas de pesquisa, dentre características funcionais e disposicionais. Geralmente, as contribuições das variáveis na explicação da variável critério são pequenas, em detrimento das variáveis ambientais, como suporte à transferência. As variáveis motivacionais têm-se mostrado as mais importantes preditoras de impacto e aprendizagem nessa linha, como afirmam Tannenbaum e Yukl (1992) e Salas e Cannon-Bowers (2001).

Neste estudo, analisa-se apenas a relação entre características demográficas dos participantes e os resultados do treinamento nos níveis de aprendizagem e comportamento no cargo, como estudo exploratório da possível influência dessas variáveis.

Seguindo a recomendação de Abbad et al. (2003), um aspecto da categoria características do treinamento, relacionado ao uso das Novas Tecnologias de Informação e Comunicação (NTICs), é analisado: a interface gráfica do curso. Trata-se do estudo do meio disponibilizado ao aluno para que adquira novos conhecimentos. Mostra-se de grande importância para os avanços em TD\&E, por ser um elemento de mediação do ensino pela Internet.

O crescente uso do ambiente da Internet para fins educacionais não tem sido acompanhado por uma avaliação sistemática em termos das conseqüências no desempenho de indivíduos, quanto à aprendizagem e aplicação de conhecimentos no trabalho. São isolados os casos de construção de instrumentos capazes de medir a funcionalidade de ambientes virtuais, apesar de já haver conhecimento sólido dos técnicos de informática sobre a concepção de softwares e a necessidade de se considerar os aspectos ergonômicos. 
As NTICs abrem um leque de possibilidades de meios para ensino a distância. No entanto trazem consigo a dificuldade de se definir a adequação dos meios aos objetivos de ensino e à elaboração de estratégias que garantam a utilização correta dos materiais e a interação com o sistema de ensino.

Scapin e Bastien (1993), a partir de amplo levantamento de indicadores de qualidade ergonômica de softwares junto a guias e revistas especializadas, descreveram oito critérios ergonômicos que deveriam nortear a elaboração e construção de softwares, que podem ser utilizados para avaliação de um ambiente de ensino a distância mediado por computador. São eles: condução, carga de trabalho, controle explícito, adaptabilidade, gestão de erros, homogeneidade/ coerência, significado dos códigos e denominações e compatibilidade. A aplicação destes critérios pode ter como consequiência a redução do número de erros e a melhoria do desempenho do usuário. Não foram encontrados dados de construção e validação estatística de instrumentos de avaliação desses critérios.

Neste estudo, serão focados os aspectos técnicos da interface do aluno com o ambiente eletrônico do curso. Interface gráfica é definida neste estudo como o local virtual, no qual o aluno tem acesso ao conteúdo do curso, bem como às ferramentas de interação, aos exercícios e às funções de suporte técnico. Com isso, pretende-se contribuir com uma avaliação da percepção dos usuários sobre esses critérios, buscando analisar sua relação com os resultados do treinamento, em termos de ganhos de aprendizagem e uso das habilidades aprendidas no contexto de trabalho.

A satisfação do aluno com o curso tem sido chamada de reação, seja aos procedimentos instrucionais do curso, à sua aplicabilidade, aos instrutores ou à interface gráfica. A revisão da produção científica nacional, realizada por BorgesAndrade e Abbad (1996), realça que o nível de avaliação mais estudado é o de reações, apesar de não haver tantos esforços em integrar outros níveis, como aprendizagem e impacto.

Como exemplo de avaliação no nível de reações, citam-se Abbad (1999) e Alves, Pasquali e Pereira (1999). Em cursos a distância, os casos são reduzidos e mais reduzidos são os estudos com avaliações sistemáticas deste nível. Dentre esses casos estão os de Carswell e Venkatesh (2002), Christensen, Anakwe e Kessler (2001), Lippert, Radhakrishna, Plank e Mitchell (2001), Porras-Hernandez (2000) e Walker (1998). Um caso isolado é o de Cheung (2000), que desenvolve um instrumento de efetividade do ensino.

Em termos metodológicos, avalia-se que os artigos levantados não apresentam critérios rigorosos em suas avaliações, havendo pouca explicação dos procedimentos de coleta e análise de dados, além de nenhum apresentar resultados 
comparativos entre níveis de avaliação. Apenas Carswell e Venkatesh (2002) comparam dados de reação e aprendizagem, porém sem medidas validadas.

Neste estudo, pretende-se cobrir parte dessas lacunas, buscando contribuir de alguma forma para a sistematização das informações colhidas em treinamentos a distância, quanto à satisfação dos participantes com relação à interface gráfica e aos resultados e à aplicabilidade do curso.

Quanto à aprendizagem, entende-se o termo como o processo no qual o indivíduo, em interação com o ambiente, adquire e retém um novo conhecimento, habilidade ou atitude, que será expressa futuramente, por meio de mudança em seu comportamento (Pantoja \& Borges-Andrade, 2002). A forma clássica de se medir o ganho de aprendizagem é por meio de pré e pós-teste, com vistas à mensuração do alcance dos objetivos de ensino traçados no planejamento do curso.

Segundo Borges-Andrade (2002), os avanços nos estudos sobre essa variável são escassos. No entanto alguns autores têm-se esforçado em utilizar medidas de aprendizagem em treinamentos a distância (Lippert et al., 2001; Walker, 1998) e presenciais (Abbad, 1999; Pantoja, Lima, \& Borges-Andrade, 2001; Sallorenzo, 2000). Os estudos analisados relatam baixíssimo poder de explicação das variáveis antecedentes sobre escores de aprendizagem, além de esta última não se configurar como preditora de impacto do treinamento no trabalho, fato também encontrado por Pantoja et al. (2001).

Já quanto ao suporte à transferência, o termo se refere às variáveis ambientais que são condição necessária para que a habilidade seja aplicada no trabalho do modo planejado. São características específicas do ambiente de trabalho que podem restringir ou facilitar a aplicação dos conhecimentos aprendidos no treinamento (Abbad, 1999).

Segundo Abbad et al. (2003), estudos em diferentes ambientes organizacionais, tipos de treinamento e amostras corroboram a importância da variável na explicação de impacto. Exemplos ilustrativos são os de Meneses e Abbad (2003) e Pantoja et al. (2001). Salas e Cannon-Bowers (2001) também relatam que os resultados sobre a importância de suporte ou clima na predição de resultados de treinamento são consistentes e passíveis de generalização. Contudo faltam estudos sobre treinamentos a distância em ambientes extra-organizacionais.

Em função da natureza do curso avaliado neste trabalho, a variável suporte à transferência deve ser redefinida. Por se tratar de um curso aberto, sem vínculo corporativo, em que o objetivo é favorecer a criação de um empreendimento, os fatores situacionais de apoio envolvem a comunidade do aluno, sua família, além 
das ações governamentais que podem facilitar ou prejudicar a abertura e manutenção de um negócio.

Peters e O'Connor (1980) abordam as restrições situacionais que podem prejudicar o desempenho no trabalho. Neste estudo, será enfocada a percepção dos participantes sobre aspectos ambientais que podem prejudicar a criação e manutenção de um negócio, após a realização de um curso a distância sobre como planejar um negócio.

As consequiências esperadas de uma ação de TD\&E passam pela satisfação com o treinamento, a aprendizagem de habilidades e pela transferência do aprendido no desempenho individual, das equipes e nas organizações. Nas pesquisas nacionais, segundo Abbad et al. (2003), o termo mais utilizado é impacto do treinamento no trabalho e compreende as noções de transferência e desempenho no trabalho. Neste trabalho, será usado o termo impacto do treinamento no trabalho, por ser considerado o conceito mais abrangente e que reflete o nível de análise aqui almejado.

Existem dois modos de se avaliar o impacto do treinamento no trabalho: em profundidade ou em amplitude. No primeiro caso, busca-se avaliar os efeitos específicos do treinamento, relacionados aos objetivos traçados no planejamento do evento ou levantados pela análise do material didático. O impacto em amplitude aborda os efeitos gerais do evento sobre o desempenho relacionado, diretamente ou não, ao conteúdo aprendido no curso. Em alguns casos, foi possível avaliar o impacto utilizando os dois tipos de medida, como colocado por Borges-Andrade (2002), tendo-se identificado altas correlações entre as medidas, fato que reforça a validade convergente das medidas.

A medida de impacto em amplitude, ou largura, pode ser utilizada para se estender a diversos contextos organizacionais (p.e., Abbad, 1999) ou para se referir às ações educacionais de uma organização específica (Borges-Andrade, 2002). Neste estudo, serão usadas medidas em profundidade e em amplitude. A medida de impacto em amplitude é construída para ser extensiva aos cursos oferecidos pela organização alvo de estudo, o Sebrae.

O impacto em amplitude, neste estudo, necessita, porém, de algumas considerações, a fim de garantir melhor compreensão por parte do leitor. Para a construção de uma medida que fosse aplicável a qualquer curso oferecido pelo Sebrae, é preciso estudar um pouco como se dão as práticas de empreendedorismo no Brasil e de que modo elas vêm sendo aplicadas. A partir de levantamento bibliográfico, não foram encontrados relatos empíricos de estudo dessa variável.

Dolabela (1999) é o grande autor brasileiro desse tema e deixa bastante claro 
que o conhecimento sobre o empreendedor ainda está em fase préparadigmática. Há pouco investimento em pesquisa sobre a possibilidade de se ensinar a ser empreendedor e sobre as características determinantes de sucesso, embora haja muitas publicações em eventos acadêmicos e criação de grande quantidade de periódicos especializados.

As noções de criatividade, iniciativa e inovação parecem ser centrais na caracterização do empreendedor. Os autores desse tema consideram que habilidades empreendedoras possam ser aprendidas e desenvolvidas e sugerem, como estratégia eficaz, identificar características de pessoas consideradas empreendedoras e compará-las às próprias características.

Com o intuito de trazer algum resultado empírico sobre o comportamento empreendedor, investigam-se neste estudo estratégias de gestão de empreendimentos por meio da medida de Impacto do Treinamento no Trabalho em Amplitude. Esta medida, portanto, visa a avaliar o perfil do empreendedor. Apresentam-se, a seguir, os objetivos a serem atingidos nesta pesquisa, bem como o modelo de investigação proposto.

\section{Objetivos de Pesquisa e Modelo de Investigação}

A partir do exposto acima, supõe-se que indivíduos mais satisfeitos com a tecnologia, que percebam que o treinamento é aplicável e trouxe resultados positivos, terão melhores escores de aprendizagem e relatarão maior impacto do treinamento no trabalho. Essa suposição é assumida tanto em termos da aplicação dos conhecimentos específicos aprendidos quanto do uso de estratégias de gerenciamento de um empreendimento.

Para examinar a suposição acima, foram estabelecidos os seguintes objetivos de pesquisa: (1) investigar as contribuições de dados demográficos e reações na explicação da variabilidade dos escores de aprendizagem dos participantes; e (2) investigar as contribuições de dados demográficos, reações e falta de suporte à transferência na explicação da variabilidade das respostas de impacto do treinamento no trabalho em profundidade e em amplitude.

Para cumprir os objetivos, elaborou-se um modelo de investigação. Trata-se de uma versão reduzida do Modelo IMPACT, de Abbad (1999). A Figura 1 apresenta a ilustração do modelo. As setas indicam a relação hipotetizada entre as variáveis. Em seguida, são definidos seus componentes. 
Figura 1: Versão Reduzida do Modelo Integrado de Avaliação do Impacto do Treinamento no Trabalho proposto por Abbad (1999)

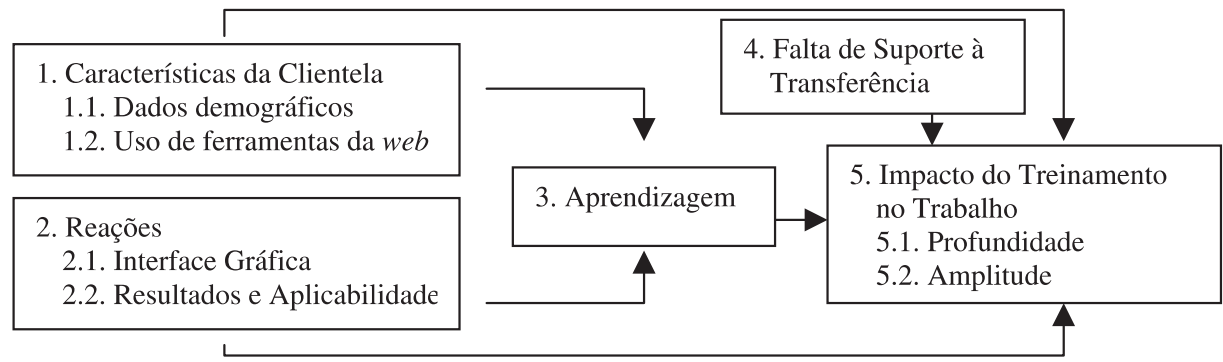

1. Características da Clientela: (1.1) dados demográficos dos participantes e (1.2) uso de ferramentas da web disponibilizadas no curso.

2. Reações: satisfação dos participantes com (2.1) a interface gráfica do curso - navegabilidade e usabilidade do ambiente na Internet e (2.2) os resultados e a aplicabilidade do curso - generalização dos conhecimentos adquiridos.

3. Aprendizagem: número de acertos dos participantes nos exercícios de fixação obrigatórios, aplicados no final de cada capítulo do curso.

4. Falta de Suporte à Transferência: opinião dos participantes a respeito de fatores ambientais, da família, da comunidade e do governo capazes de prejudicar a abertura e manutenção de um empreendimento.

5. Impacto do Treinamento no Trabalho: avaliação dos participantes sobre os efeitos do treinamento, medidos (5.1) em profundidade - aplicação dos desempenhos traçados nos objetivos de ensino no ambiente de trabalho e (5.2) em amplitude - emprego de estratégias de gerenciamento de um empreendimento.

\section{MÉTOdo}

Nesta seção, faz-se uma breve descrição da organização alvo e do treinamento avaliado. Em seguida, descreve-se a amostra, apresentam-se os instrumentos de medida e os procedimentos de coleta e análise dos dados da pesquisa.

O Sebrae (Serviço Brasileiro de Apoio às Micro e Pequenas Empresas), voltado para o fomento e difusão de programas e projetos que visam à promoção e ao fortalecimento das micro e pequenas empresas, busca primordialmente promover o desenvolvimento sustentável do país, por meio de estratégias que levem ao 
crescimento do empreendedorismo como forma de fonte de renda alternativa para a população brasileira.

Avaliou-se o curso Iniciando um Pequeno Grande Negócio (IPGN), oferecido gratuitamente, em nível nacional, mediado por Internet e material impresso. O objetivo geral do curso é permitir que o aluno seja capaz de elaborar um Plano de Negócio - documento escrito que especifica os principais fatores necessários à criação de uma empresa. Destina-se a empreendedores que desejam abrir um negócio ou àqueles que já possuem um negócio e necessitam de informações adicionais para melhorar os resultados do empreendimento. Recomenda-se escolaridade de segundo grau completo e acesso regular à Internet.

O conteúdo programático é apresentado em cinco módulos seqüenciais, cada qual composto por capítulos em número de dois a cinco, acessíveis ao aluno no portal eletrônico do curso e/ou por meio de download do material. São eles: (1) perfil empreendedor; (2) identificando oportunidades de negócios; (3) análise de mercado; (4) concepção dos produtos e serviços; e (5) análise financeira. Além dos materiais e exercícios, o portal contém os seguintes recursos eletrônicos de interação (aluno-aluno; aluno-tutor; aluno-tutor-material): tutoria ativa, lista de discussão, mural de notícias, chat, troca de mensagens.

A duração prevista para realização do curso é 40 horas, distribuídas em 60 dias. São propostas atividades no final de cada capítulo, de caráter optativo, e um exercício de fixação obrigatório, com questão de múltipla escolha, que permite o acesso ao capítulo seguinte.

Compuseram a amostra do estudo alunos do IPGN matriculados nas turmas oferecidas de $1^{\circ}$ de maio a 6 de agosto de 2002. Do total de 21.273 alunos matriculados no IPGN no período avaliado, 11.597 (52,9\%) concluíram o curso. O retorno geral dos instrumentos de reação foi de 4.323 (24,12\% para a população de inscritos e 37,28\% para os concluintes) e, o de impacto, de 1.622 (8,45\% para a população e $13,99 \%$ para os concluintes).

Do conjunto de respostas recebidas, foram expurgados os registros duplicados e vazios, chegando-se então a um total de 4.078 registros que possuíam resposta a pelo menos um instrumento. Com o intuito de garantir o máximo de casos nas análises, foi necessário criar um arquivo de dados para cada modelo testado de relacionamento entre variáveis. O Modelo 1, com variável critério Escores de Aprendizagem, apresentou 1.659 casos; o Modelo 2 (Impacto em Profundidade), 340 casos; e o Modelo 3 (Impacto em Amplitude), 355 casos.

Algumas informações sobre o perfil de inscritos no período estudado foram disponibilizadas pelo Sebrae. Percebeu-se que as amostras dos três modelos de 
regressão testados, assim como da população de concluintes do curso, apresentavam perfil bastante semelhante. Trata-se de alunos essencialmente do sexo masculino, com idade média de 34 anos (desvio padrão $=10$ ), escolaridade superior incompleta e residentes na região Sudeste do país. Houve uso pouco frequiente das ferramentas da web disponibilizadas: a maioria não enviou mensagem à seção tira-dúvida, enviou de 1 a 10 mensagens para a lista de discussão, não acessou o chat e acessou o ambiente do curso de 11 a 30 vezes durante os 60 dias previstos.

Nota-se ainda que se trata de amostras de participantes que, em sua maioria, não possuem um empreendimento, consideram-se experientes no uso da Internet, não participaram anteriormente de curso a distância, trabalham em empresa privada, possuem maior interesse no setor de serviços e inscreveram-se no curso para aprender a elaborar um plano de negócio.

A semelhança das amostras com a população é muito positiva, pois fornece certo respaldo aos resultados das análises realizadas. Apesar de se tratar de amostras muito reduzidas em relação à população (chega-se a ter menos de $2 \%$ de representação da população nas amostras), os indivíduos têm perfil equiparável, o que permite afirmar com menos incerteza que os resultados refletem características dos indivíduos inscritos no curso IPGN.

No nível de reação, foram utilizados instrumentos de medida para avaliação de reação a interface gráfica do curso e a resultados e aplicabilidade do curso. No nível de impacto, foram utilizados instrumentos de avaliação do impacto do treinamento no trabalho (em profundidade e em amplitude) e falta de suporte à transferência. Além destas medidas, incluem-se dados demográficos e pessoais sobre os participantes, descritos acima, e escores de aprendizagem, fornecidos pelo Sebrae.

Todos os instrumentos de natureza perceptual foram validados estatisticamente com a mesma população de participantes do IPGN utilizada nesta pesquisa. Todos os instrumentos apresentaram estruturas unifatoriais, agrupando todos os itens inicialmente elaborados. Alguns deles permitem o uso de escalas com duas ou três dimensões, como foi o caso das medidas de Falta de Suporte à Transferência e Impactos no Trabalho. Por serem mais parcimoniosas, optou-se pelo uso das escalas unidimensionais. A seguir, apresentam-se os principais resultados das validações.

O instrumento de Reação à Interface Gráfica compõe-se de 15 itens, associados a uma escala de intensidade de 11 pontos ( 0 - péssimo; 10 - excelente). A validação estatística indica alfa de Cronbach de 0,95 e 57,46\% de variância explicada. O instrumento de Reação a Resultados e Utilidade inclui 7 itens, vinculados a uma 
escala de concordância de 11 pontos (0 - discordo totalmente; 10 - concordo totalmente). A validação estatística indica alfa de Cronbach de 0,88 , com explicação de $54,30 \%$ da variância total das respostas.

Os Escores de Aprendizagem são utilizados como o somatório de acertos nos exercícios de fixação, a serem respondidos ao final de cada capítulo do curso.

A Escala de Falta de Suporte à Transferência inclui 15 itens associados a uma escala que varia de 0 (nada) a 10 (totalmente). $O$ instrumento validado apresenta alfa de Cronbach de 0,91 e explicação de 40,93\% da variância total das respostas aos itens.

O Impacto do Treinamento no Trabalho (em Profundidade) foi medido por uma escala de 24 itens vinculados a escala de freqüência de 0 (nunca) a 10 (sempre). Os índices psicométricos apontam alfa de Cronbach de 0,96 e 52,01\% de variância explicada pelo fator. O instrumento inclui mais um item, Elaborei meu plano de negócio, por se tratar do objetivo final planejado pelo curso, porém sem obrigatoriedade de ser realizado. Este item foi respondido com opções 1 (sim) e 2 (não). O instrumento de Impacto do Treinamento no Trabalho (em Amplitude) agrupa 37 itens associados a uma escala de frequiência de 11 pontos, com ancoragem nos pontos extremos ( 0 - nunca; 10 - sempre). $\mathrm{O}$ instrumento validado estatisticamente apresenta alfa de Cronbach de 0,97 e 54,45\% de variância explicada pelo fator identificado.

A coleta de dados ocorreu em dois momentos. No último dia de curso, coletaram-se dados de reação à interface gráfica e a resultados e aplicabilidade, além de serem solicitados dados pessoais dos alunos. No segundo momento, de 42 a 105 dias após o término do curso, foram coletados os dados de falta de suporte à transferência e impacto do treinamento no trabalho, medido em profundidade e em largura.

Nos dois momentos de avaliação, os alunos receberam um e-mail contendo o link para acesso aos questionários, os quais estavam disponíveis na Internet. Os respondentes deviam passar por três páginas até concluir o preenchimento dos questionários, quando recebiam uma mensagem de agradecimento, informando que os dados haviam sido enviados com sucesso. As análises foram realizadas no programa SPSS for Windows, versão 10.0.

Foram realizadas análises estatísticas exploratórias para limpeza de cada arquivo de dados, observando-se distribuição das variáveis, dados extremos e dados omissos. O tratamento escolhido para os dados omissos foi do tipo pairwise, por não haver mais de 5\% de casos em cada variável estudada. Em relação aos casos extremos multivariados, usou-se o método de cálculo da distância 
Mahalanobis, com $\mathrm{p}<0,001$. As análises de regressão foram realizadas com e sem a presença desses casos. Por não haver diferença na ordem de importância dos coeficientes $(\beta)$ e no coeficiente de regressão múltipla $\left(\mathrm{R}^{2}\right)$, optou-se por manter todos os casos extremos nas análises, garantindo maior generalidade dos resultados.

As análises inferenciais escolhidas foram a regressão múltipla padrão e stepwise. De acordo com o critério sugerido por Tabachnick e Fidell (2001), de acordo com o número de variáveis antecedentes, seriam necessários 280 casos válidos para realizar as análises com confiabilidade. Assim, todos os modelos tiveram quantidade de casos válidos suficiente.

Foram testados três modelos distintos de regressão, com três variáveis critério: (1) Escores de Aprendizagem, (2) Impacto do Treinamento no Trabalho (em Profundidade) e (3) Impacto do Treinamento no Trabalho (em Amplitude). As variáveis antecedentes foram definidas em função da correlação existente com a variável critério, a fim de evitar um modelo inflacionado e a ocorrência de erro tipo I.

\section{Resultados}

Para as variáveis medidas pelos instrumentos validados, foram realizadas médias aritméticas de todos os itens correspondentes ao fator identificado na análise fatorial. São apresentados, portanto, os resultados das análises de relacionamento entre variáveis.

O arquivo de dados para avaliação do modelo de predição dos Escores de Aprendizagem continha 1.659 casos válidos. As variáveis antecedentes que entraram nas análises de regressão, isto é, as que apresentaram correlações bivariadas significativas com o critério, foram: Reação à Interface Gráfica, Reação a Resultados e Aplicabilidade, Número de Dúvidas Enviadas ao Tira-Dúvidas, Número de Acessos ao Mural de Notícias, Número de Mensagens Enviadas à Lista de Discussão e Número de Acessos ao Chat.

A Tabela 1 apresenta os resultados da análise de regressão múltipla padrão. As correlações entre as variáveis, os coeficientes de regressão padronizados $(\beta)$ e não padronizados (B), a constante, bem como o $R^{2}$ e o $R^{2}$ ajustado estão disponibilizados. 
Tabela 1: Regressão Múltipla Padrão para o Modelo 1

\begin{tabular}{lccccccc}
\hline \hline \multicolumn{1}{c}{ Variável } & Aprend (VD) & tira-dúvida & mural & lista & chat & ambiente & resultado \\
\hline tira-dúvida & 0,05 & & & & & & \\
mural & 0,16 & 0,10 & & & & & \\
lista & 0,06 & 0,15 & 0,24 & & & & \\
chat & 0,19 & 0,12 & 0,37 & 0,20 & & & \\
ambiente & 0,05 & $-0,03$ & 0,01 & $-0,01$ & 0,00 & & \\
resultado & 0,13 & 0,01 & 0,03 & 0,03 & 0,01 & 0,53 & \\
\hline $\mathrm{B}$ & 0,07 & $0,00^{*}$ & 0,00 & $0,01 *$ & $-0,04$ & $0,28^{*}$ \\
$\beta$ & 0,02 & 0,10 & 0,00 & 0,15 & $-0,02$ & 0,13 \\
$\mathrm{sr}^{2}$ & 14,57 & 0,00 & 0,01 & 0,00 & 0,02 & 0,00 & 0,01 \\
$\mathrm{X}$ & 2,66 & 0,89 & 47,37 & 2,52 & 35,87 & 8,56 & 8,39 \\
DP & 52,50 & 4,27 & 38,87 & 1,19 & 1,27 \\
\hline \multicolumn{7}{l}{ Constante $=11,9 ; R^{2}=0,06 ; R^{2}$ (ajustado) $=0,06 \mathrm{e} ; R=0,25^{*} ; * \mathrm{p}<0,01$} \\
\end{tabular}

Três variáveis apresentaram contribuição significativa para a explicação da variável critério. Foram elas: Acesso ao Mural, Acesso ao Chat e Reação a Resultados e Aplicabilidade. Todas as seis variáveis antecedentes testadas no modelo contribuíram com explicação de $6 \%$ da variável critério Escores de Aprendizagem.

Os resultados indicam, assim, que os participantes que tiveram maior freqüência de participação nos chats, que acessaram mais vezes o ambiente do curso e que consideraram o curso aplicável e generalizável obtiveram maiores escores de aprendizagem.

No modelo de avaliação do Impacto do Treinamento (em Profundidade), em arquivo com 340 casos, são inseridas como variáveis antecedentes a Reação à Interface Gráfica, a Reação a Resultados e Aplicabilidade, a Falta de Suporte à Transferência, a Região Geográfica e a Elaboração do Plano de Negócio. Cumprindo os pressupostos da análise de regressão, a variável Região Geográfica foi dicotomizada nas categorias 1 (região Sudeste) e 0 (demais regiões). A Tabela 2 apresenta os resultados da regressão múltipla padrão deste modelo.

Tabela 2: Regressão Múltipla Padrão para o Modelo 2

\begin{tabular}{lcccccc}
\hline \multicolumn{1}{c}{ Variável } & Prof (VD) & região & plano & ambiente & resultado & suporte \\
\hline região & $-0,16$ & & & & & \\
plano & $-0,26$ & 0,08 & & & & \\
ambiente & 0,27 & 0,00 & 0,01 & & & \\
resultado & 0,48 & $-0,09$ & $-0,14$ & 0,60 & & \\
suporte & 0,29 & $-0,06$ & $-0,05$ & 0,04 & 0,04 & \\
\hline $\mathrm{B}$ & & $-0,29$ & $-0,67^{*}$ & $-0,007$ & $0,63^{*}$ & $0,22^{*}$ \\
$\beta$ & & $-0,08$ & $-0,18$ & $-0,005$ & 0,44 & 0,26 \\
$\mathrm{sr}^{2}$ & & 0,00 & 0,03 & 0,00 & 0,12 & 0,06 \\
$\mathrm{X}$ & 7,59 & - & - & 8,66 & 8,53 & 6,23 \\
DP & 1,66 & - & - & 1,12 & 1,15 & 1,98 \\
\hline \hline
\end{tabular}


Três variáveis tiveram contribuições individuais $\left(\mathrm{sr}^{2}\right)$ significativas para a explicação de Impacto do Treinamento no Trabalho em Profundidade: Elaboração do Plano de Negócio, Reação a Resultados e Aplicabilidade e Falta de Suporte à Transferência. As cinco variáveis antecedentes explicaram 34\% (33\% ajustado) da variância de impacto em profundidade percebido.

No modelo de avaliação do Impacto do Treinamento (em Amplitude), as variáveis que apresentaram correlação significativa com a variável critério foram idênticas ao Modelo 2. Novamente, a variável Região Geográfica foi codificada em duas categorias. A Tabela 3 apresenta os resultados da análise.

Tabela 3: Regressão Múltipla Padrão para o Modelo 3

\begin{tabular}{lcccccc}
\hline \multicolumn{1}{c}{ Variável } & Prof (VD) & região & plano & ambiente & resultado & suporte \\
\hline região & $-0,13$ & & & & & \\
plano & $-0,20$ & 0,08 & & & & \\
ambiente & 0,32 & 0,00 & 0,02 & & & \\
resultado & 0,47 & $-0,09$ & $-0,13$ & 0,59 & & \\
suporte & 0,27 & $-0,05$ & $-0,03$ & 0,03 & 0,03 & \\
\hline $\mathrm{B}$ & & $-0,21$ & $-0,46^{*}$ & 0,11 & $0,52^{*}$ & $0,19^{*}$ \\
$\beta$ & & 0,07 & $-0,14$ & 0,08 & 0,40 & 0,25 \\
$\mathrm{sr}^{2}$ & & 0,00 & 0,02 & 0,00 & 0,09 & 0,06 \\
$\mathrm{X}$ & 8,14 & - & - & 8,65 & 8,53 & 6,25 \\
DP & 1,51 & - & - & 1,12 & 1,15 & 1,97 \\
\hline \hline
\end{tabular}

As três variáveis com explicação significativa do critério foram: Elaboração de Plano de Negócio, Reação a Resultados e Aplicabilidade e Falta de Suporte à Transferência. As cinco variáveis antecedentes explicaram 31\% (30\% ajustado) da variância total das respostas de Impacto do Treinamento no Trabalho (em Amplitude).

Nos Modelos 2 e 3, os participantes relatam maior impacto do treinamento no trabalho (em profundidade e em amplitude), quando reagem positivamente aos resultados e à aplicabilidade do curso, quando percebem falta de suporte à transferência e quando elaboram o plano de negócio.

Como havia casos suficientes para realizar análise de regressão múltipla com método stepwise, para os três modelos apresentados, esse procedimento foi seguido para corroborar os resultados encontrados no primeiro método. Os resultados praticamente não diferiram em todas as análises. As mesmas variáveis antecedentes tiveram contribuição significativa na explicação das variáveis critério, com ordem de entrada e contribuições parciais semelhantes. O coeficiente de regressão múltipla $\mathrm{R}^{2}$ também foi igual para os dois métodos. A semelhança relatada fortalece os resultados encontrados. 


\section{Discussão}

Esta seção aborda os resultados encontrados na pesquisa, por meio do estabelecimento de relações com a revisão de literatura, e identifica problemas teóricos, lacunas e falhas na pesquisa. Implicações teóricas, metodológicas e práticas são igualmente enfocadas.

O Modelo 1 apresentou resultados semelhantes aos da literatura (Abbad, 1999; Pantoja et al., 2001), em que o poder preditivo das variáveis é muito baixo. Nenhuma das variáveis aqui estudadas foi apontada na literatura como preditora de aprendizagem, em cursos presenciais e a distância. Esses resultados, no entanto, estão longe de ser conclusivos, principalmente pela medida de aprendizagem utilizada, que não parece ser fidedigna em face dos objetivos traçados. Sugerese, além de uma medida que corresponda aos objetivos de desempenho, o uso de medidas de aprendizagem perceptuais e não perceptuais, com pré e pós-teste.

Mesmo com tantos avanços em avaliação de treinamento, muito pouco se sabe sobre as variáveis que predizem a aprendizagem. Este estudo não corroborou os achados de Warr e Birdi (1998), Abbad (1999) e Pantoja et al. (2001), quanto ao poder preditivo de dados demográficos, como idade e nível de instrução. Sugerese que variáveis motivacionais também sejam estudadas.

Nos Modelos 2 e 3, as mesmas variáveis apresentaram contribuição significativa na explicação da variável critério. Foram elas, na ordem de magnitude das contribuições individuais: Reação aos Resultados e Aplicabilidade, Falta de Suporte à Transferência e Elaboração de Plano de Negócio.

A primeira variável encontra suporte parcial em alguns estudos que a utilizaram em avaliação de treinamentos presenciais, embora com medidas não totalmente equivalentes (Abbad, 1999; Sallorenzo, 2000). Em geral, no Brasil, tem-se encontrado apoio para a importância das reações ao treinamento como preditoras de impacto do treinamento no trabalho; mas, como sugere Sallorenzo (2000), os resultados ainda não são conclusivos e são necessárias mais pesquisas que façam uso dessas variáveis.

Todavia a variável Reação à Interface Gráfica não se mostrou preditora de Impacto, fato inesperado no momento de planejamento da pesquisa. Percebe-se, porém, que a variável apresentou correlação alta e significativa com a medida de impacto, tanto em profundidade quanto em largura. $\mathrm{O}$ motivo para não ter permanecido na equação final das análises de regressão pode estar no fenômeno de redundância identificado, de acordo com os indicadores de Abbad e Torres (2002). O resultado sugere, portanto, que Reação à Interface Gráfica não pode 
ser descartada e deve ser analisada em outros contextos, mesmo porque seu estudo sugere uma integração com a Ergonomia, área do conhecimento dificilmente associada aos estudos que fazem uso da Psicologia Instrucional.

A presença da variável Falta de Suporte à Transferência como preditora corrobora achados de diversos estudos, como os de Pantoja et al. (2001), Meneses e Abbad (2003) e Lacerda e Abbad (2003). Porém, nesta pesquisa, a magnitude do relacionamento foi menor e inversa. O resultado, que poderia ser entendido como negativo, deve ser relativizado, uma vez que o curso avaliado tem certas peculiaridades que podem ter influenciado as relações encontradas entre variáveis.

Os resultados das análises indicaram que os respondentes que percebem menos suporte à abertura e manutenção de um empreendimento obtêm escores mais altos de impactos no trabalho. O relacionamento negativo entre medidas de suporte à transferência e impacto não foi encontrado em relatos anteriores. $\mathrm{O}$ resultado poderia ser explicado pelo fato de o contexto do empreendedorismo valorizar desafios. A falta de suporte, sendo entendida como desafio, pode ter sido valorizada pelos respondentes e vista como estímulo à utilização dos novos conhecimentos.

A terceira variável que explicou os Impactos no Trabalho foi a Elaboração de Plano de Negócio. A variável parece ser realmente importante, tanto para o planejamento (impacto em profundidade), quanto para a administração (impacto em amplitude) de um negócio. O resultado indica que o Sebrae oferece um curso que ensina uma habilidade que realmente é necessária ao empreendedorismo. No entanto, no IPGN, a atividade não é obrigatória. Uma ação que poderia aumentar os resultados de impacto, ou aumentar o número de participantes que abrem um negócio, seria investir em estratégias que estimulem os alunos a elaborarem o documento.

Existe alta correlação entre as duas medidas de Impacto no Trabalho. Há indicadores de que são medidas distintas, porém ainda é preciso discutir que tipo de relação pode ser estabelecida entre elas. A hipótese levantada aqui é de que os participantes que relataram alta freqüência no uso das habilidades descritas na escala de Impacto em Amplitude já as possuíam antes da realização do treinamento. Para verificar essa hipótese, sugere-se a inclusão, em pesquisas futuras, de uma medida de pré-teste de empreendedorismo, quando poderiam ser identificadas características pessoais que definam o empreendedor, além de estratégias comportamentais para enfrentar dificuldades e barreiras à abertura de um negócio.

Um dos aspectos que mais preocupam os educadores quanto à difusão da educação a distância refere-se aos índices de evasão. Este é tema que necessita 
investigação. Não se tem informação consistente sobre as variáveis que interferem na manutenção de um indivíduo em curso oferecido via web. Algumas análises preliminares aqui realizadas indicaram diferenças, entre concluintes e não concluintes, sobre o uso de ferramentas da web, o que poderia ser um indicador de permanência ou evasão dos participantes. Outras variáveis, em especial as de caráter motivacional, deveriam ser estudadas, uma vez que o estudo a distância fica muito centrado no próprio aluno.

\section{Considerações Finais}

Quanto às limitações do estudo, alguns aspectos metodológicos devem ser destacados. O baixo índice de retorno dos questionários deveu-se a falhas na programação dos instrumentos, na hospedagem deles na Internet e nos arquivos de dados eletrônicos, além de problemas de queda de conexão dos computadores pessoais dos usuários. O delineamento da coleta de dados, todavia, também apresentou falhas que prejudicaram o retorno dos questionários. $\mathrm{O}$ custo para $\mathrm{o}$ usuário participar da pesquisa também parece ter influenciado o recebimento dos dados. Apesar dos problemas apontados na metodologia de coleta de dados, não se considera que este método seja desprezível. Em toda coleta de dados, geralmente serão necessários ajustes metodológicos, fato que não pode impedir a realização da pesquisa. Recomenda-se apenas cautela ao abordar os achados desta pesquisa em outros contextos, por se tratar de curso aberto, gratuito e não corporativo.

De modo geral, os resultados das análises de relacionamento entre variáveis não trazem respostas, apenas caminhos que ainda precisam ser longamente trilhados. Foram identificadas variáveis preditoras de resultados de treinamento nos níveis de aprendizagem e impacto do treinamento no trabalho; porém seus poderes preditivos, em todos os modelos, foram baixos. Este fato apenas sugere que são muitas as possíveis variáveis influentes nas ações de EaD.

Por fim, esta pesquisa atende à recomendação feita nas últimas revisões de literatura sobre treinamento e desenvolvimento (Abbad et al., 2003; Salas \& Cannon-Bowers, 2001), qual seja a de avaliar um curso oferecido a distância. Almeja-se dar início ao estudo mais aprofundado das novas mídias em ações educacionais corporativas, buscando a consolidação de um saber que possa nortear intervenções e otimizar os altos investimentos em TD\&E.

\section{Artigo recebido em 28.03.2004. Aprovado em 17.07.2004.}




\section{ReferênCIAS Bibliográficas}

Abbad, G. (1999).

Um modelo integrado de avaliação de impacto do treinamento no trabalho - IMPACT. Tese de Doutorado, Instituto de Psicologia, Universidade de Brasília, Brasília.

Abbad, G.,

Pilati, R., \&

Pantoja, M. J. B. (2003, julho/agosto/ setembro).

Avaliação de treinamento: análise da literatura e agenda de pesquisa. Revista de Administração, 38(3), 205218.

Abbad, G., \&

Torres, C. V. (2002).

Regressão múltipla stepwise e hierárquica em Psicologia Organizacional: aplicações, problemas e soluções. Estudos de Psicologia, 7(especial), 19-29.

Alliger, G. M., \&

Janak, E. A. (1989, Summer).

Kirkpatrick's levels of training criteria: thirty years later. Personnel Psychology, 42(2), 331-342.

Alves, A. R.,

Pasquali, L., \&

Pereira, M. A. M. (1999, janeiro/março).

Escala de satisfação com o treinamento-ESAST/Telebrás/UnB. Revista de Administração de Empresas, 39 (1), 25-30.

Belloni, M. L. (1999).

Educação a distância. Campinas, SP: Autores Associados.
Borges-Andrade, J. E. (1982).

Avaliação somativa de sistemas instrucionais: integração de três propostas. Tecnologia Educacional, 11(46), 29-39.

Borges-Andrade, J. E. (1997).

Treinamento de pessoal: em busca de conhecimento e tecnologia relevantes para as organizações brasileiras. In: A. Tamayo, J. E. Borges-Andrade, \& W. Codo (Orgs.). Trabalho, Organizações e Cultura. (pp.116-133). São Paulo: Cooperativa de Autores Associados.

Borges-Andrade, J. E. (2002).

Desenvolvimento de medidas em avaliação de treinamento. Estudos de Psicologia, 7(especial), 31-43.

Borges-Andrade, J. E., \&

Abbad, G. (1996, abril/junho).

Treinamento no Brasil: reflexões sobre suas pesquisas. Revista de Administração, 31(2), 112-125.

Carswell, A. D., \&

Venkatesh, V. (2002, May).

Learner outcomes in an asynchronous distance education environment. International Journal of Human-Computer Studies, 56(5), 475-494.

Cheung, D. (2000).

Evidence of a single second-order factor in student ratings of teaching effectiveness. Structural Equation Modeling: a Multidisciplinary Journal, 7(3), 442-460. 
Christensen, E. W.,

Anakwe, U. P., \&

Kessler, E. H. (2001).

Receptivity to distance learning: the effect of technology, reputation, constraints, and learning preferences. Journal of Research on Computing in Education, 33(3), 263-279.

Dolabela, F. (1999).

Oficina do empreendedor. São Paulo: Cultura.

Hamblin, A. C. (1978).

Avaliação e controle de treinamento. São Paulo: McGraw-Hill do Brasil.

Kirkpatrick, D. L. (1976).

Evaluation of training. In: R. L. Craig. Training and development handbook. (2nd ed., pp.18.1-18.27).

New York: McGraw-Hill.

Lacerda, E. R. M., \&

Abbad, G. (2003).

Impacto do treinamento no trabalho: investigando variáveis motivacionais e organizacionais como suas preditoras. Revista de Administração Contemporânea, 7(4), 77-96.

Lippert, R. M.,

Radhakrishna, R.,

Plank, O., \&

Mitchell, C. C. (2001)

Using different evaluation tools to assess a regional Internet inservice training. International Journal of Instructional Media, 28(3), 237-248.
Maggio, M. (2001).

O tutor na educação a distância. In: E. Litwin (Org.). Educação a distância - temas para o debate de uma nova agenda educativa (pp. 93110). Porto Alegre: Artmed.

Meister, J. C. (1999).

Educação corporativa. São Paulo: Makron Books.

Meneses, P. P. M., \&

Abbad, G. (2003).

Preditores individuais e situacionais de auto e heteroavaliação de impacto do treinamento no trabalho. Revista de Administração Contemporânea, 7(especial), 185-204.

Pantoja, M. J. B, \&

Borges-Andrade, J. E. (2002).

Uma abordagem multinível para o estudo da aprendizagem e transferência nas organizações. Anais do Encontro Nacional dos Programas de Pós-Graduação em Administração, Salvador, BA, 26.

Pantoja, M. J. B., Lima, S. M. V., \&

Borges-Andrade, J. E. (2001, abril/junho). Avaliação de impacto de treinamento na área de reabilitação: preditores individuais e situacionais. Revista de Administração, 36(2), 46-56.

Peters, L. H., \&

O’Connor, E. J. (1980, July).

Situational constraints and work outcomes: the influences of a frequently overlooked construct. Academy of Management Review, 5(3), 391-397. 
Porras-Hernandez, L. H. (2000).

Student variables in the evaluation of mediated learning environments. Distance Education: an International Journal, 21(2), 385403.

Salas, E., \&

Cannon-Bowers, J. (2001).

The science of training: a decade of progress. Annual Review of Psychology, 52, 471-499.

Sallorenzo, L. H. (2000).

Avaliação de Impacto do Treinamento no Trabalho: analisando e comparando modelos de predição. Dissertação de Mestrado, Instituto de Psicologia, Universidade de Brasília, Brasília, DF.

Scapin, D. L., \&

Bastien, J. M. C. (1993).

Critères ergonomiques pour l'évaluation d'interfaces utilisateurs. (Rapports Techniques, RT-0156), Le Chesnay, França: INRIA Rocquencourt.
Tabachnick, B. G., \&

Fidell, L. S. (2001).

Using Multivariate Statistics (4th ed.). New York: HarperCollins.

Tannenbaum, S. I., \&

Yukl, G. (1992, January).

Training and development in work organizations. Annual Review of Psychology, 43, 399-441.

Walker, S. (1998).

The value of building skills with online technology: online training costs and evaluation at the Texas Natural Resource Conservation Commission. In: D. A. Schreiber, Z. L. Berge, (Eds.). Distance Training (pp. 270-286). San Francisco, CA: Jossey Bass.

Warr, P., \&

Birdi, K. (1998, September).

Employee age and voluntary development activity. International Journal of Training and Development, 2(3), 190-204. 